Research Paper

\title{
Inverse Association between Sodium Channel-Blocking Antiepileptic Drug Use and Cancer: Data Mining of Spontaneous Reporting and Claims Databases
}

\author{
Mitsutaka Takada ${ }^{\bowtie}$, Mai Fujimoto, Haruka Motomura, Kouichi Hosomi \\ Division of Clinical Drug Informatics, School of Pharmacy, Kinki University, 3-4-1, Kowakae, Higashi-osaka, Osaka, 577-8502, Japan. \\ $\triangle$ Corresponding author: Mitsutaka Takada, PhD, Division of Clinical Drug Informatics, School of Pharmacy, Kinki University, 577-8502, 3-4-1, Kowakae, \\ Higashi-osaka, Osaka, 577-8502, Japan. Telephone number: +81-6-6721-2332, Fax number: +81-6-6730-1394, E-mail address: takada@phar.kindai.ac.jp \\ (c) Ivyspring International Publisher. Reproduction is permitted for personal, noncommercial use, provided that the article is in whole, unmodified, and properly cited. See \\ http://ivyspring.com/terms for terms and conditions.
}

Received: 2015.09.13; Accepted: 2015.11.27; Published: 2016.01.01

\begin{abstract}
Purpose: Voltage-gated sodium channels (VGSCs) are drug targets for the treatment of epilepsy. Recently, a decreased risk of cancer associated with sodium channel-blocking antiepileptic drugs (AEDs) has become a research focus of interest. The purpose of this study was to test the hypothesis that the use of sodium channel-blocking AEDs are inversely associated with cancer, using different methodologies, algorithms, and databases.

Methods: A total of 65,146,507 drug-reaction pairs from the first quarter of 2004 through the end of 2013 were downloaded from the US Food and Drug Administration Adverse Event Reporting System. The reporting odds ratio (ROR) and information component (IC) were used to detect an inverse association between AEDs and cancer. Upper limits of the $95 \%$ confidence interval $(\mathrm{Cl})$ of $<1$ and $<0$ for the ROR and IC, respectively, signified inverse associations. Furthermore, using a claims database, which contains 3 million insured persons, an event sequence symmetry analysis (ESSA) was performed to identify an inverse association between AEDs and cancer over the period of January 2005 to May 2014. The upper limit of the $95 \% \mathrm{Cl}$ of adjusted sequence ratio (ASR) $<1$ signified an inverse association.

Results: In the FAERS database analyses, significant inverse associations were found between sodium channel-blocking AEDs and individual cancers. In the claims database analyses, sodium channel-blocking AED use was inversely associated with diagnoses of colorectal cancer, lung cancer, gastric cancer, and hematological malignancies, with ASRs of 0.72 ( $95 \% \mathrm{Cl}: 0.60-0.86$ ), $0.65(0.51-0.81), 0.80(0.65-0.98)$, and $0.50(0.37-0.66)$, respectively. Positive associations between sodium channel-blocking AEDs and cancer were not found in the study.

Conclusion: Multi-methodological approaches using different methodologies, algorithms, and databases suggest that sodium channel-blocking AED use is inversely associated with colorectal cancer, lung cancer, gastric cancer, and hematological malignancies.
\end{abstract}

Key words: Voltage-gated sodium channels

\section{Introduction}

Voltage-gated sodium channels (VGSCs) are drug targets for the treatment of epilepsy [1]. Recent$1 \mathrm{y}$, the expression of VGSCs has been identified in a number of major cancers [2,3], and many studies have indicated that VGSCs promote in vitro cellular behaviors associated with metastasis, including migration and invasion [4-9]. VGSCs are up-regulated in human metastatic disease, and VGSC activity potentiates metastatic cell behavior $[6,10,11]$. Therefore, blockage of these channels may be effective for treatment of cancer. Cancer is a leading cause of death worldwide, and metastasis is a major concern with cancer treatment, as metastatic cancer is rarely responsive to treatment. Inhibition of tumor growth and 
metastasis is the most practical goal for those patients who are unable to tolerate radical surgery or are deemed unsuitable for surgery. Therefore, better strategies for prevention of metastasis are desired. In recent years, the focus has been on the role of ion channels in the development and progression of cancer. A few mechanisms have been suggested for the role of VGSCs in migration and invasion of cancer cells. The effects of VGSCs have been associated with regulation of $\mathrm{pH}$, gene expression and intracellular calcium levels $[5,12,13]$. However, the mechanism(s) regulating functional VGSC expression in cancer cells remains unknown.

Antiepileptic drugs (AEDs) including phenytoin, carbamazepine, lamotrigine, topiramate, valproic ac$\mathrm{id}$, and ethotoin are representative sodium channel-blocking drugs. Therefore, use of AEDs is expected to delay the development of metastasis and thus prolong survival in patients with cancer. However, the relationship between sodium channel-blocking AEDs and survival of cancer patients has remains unclear. Recently, a cohort study using a medical database comprising 100,000 patients diagnosed with breast, colorectal or prostate cancer was designed to test the hypothesis that sodium channel-blocking drugs delay the development of metastasis and thus prolong survival of cancer patients [14]. However, at present, no definitive evidence exists to support this hypothesis.

In recent years, data mining utilizing different methodologies, algorithms, and databases has been used to identify risk signals within medical databases, including spontaneous adverse drug reaction databases, claim databases, and prescription databases. We applied these methodologies and algorithms to the detection of inverse signals of cancer associated with sodium channel-blocking AED use.

\section{Methods}

\section{Data from the US Food and Drug Administra- tion (FDA) Adverse Event Reporting System (FAERS)}

The FAERS is a computerized information database designed to support the FDA's post-marketing safety surveillance program for all approved drugs and therapeutic biological products. The system contains all reports of adverse events reported spontaneously by health care professionals, manufacturers, and consumers worldwide. The FAERS consists of seven datasets that include patient demographic and administrative information (file descriptor DEMO), drug and biological information (DRUG), adverse events (REAC), patient outcomes (OUTC), report sources (RPSR), start of drug therapy and end dates
(THER), and indications for use/diagnosis (INDI). Unique identification numbers for each FAERS report allow linkage of all information from different files. The raw data from the FAERS database can be downloaded freely from the FDA website (http://www.fda.gov/Drugs/InformationOnDrugs/ ucm135151.htm).

The present study included FAERS data from the first quarter of 2004 through the end of 2013. A total of $4,866,160$ reports were obtained. Reports with a common case number were identified as duplicate reports and were excluded from the analyses. Finally, a total of $65,146,507$ drug-reaction pairs were identified among 4,081,582 reports. The preferred terms (PTs) of the Medical Dictionary for Regulatory Activities (MedDRA ${ }^{\circledR}$ version 17.0) were used to classify adverse events.

\section{Identifying AEDs and cancers}

The FAERS permits the registration of arbitrary drug names including trade and generic names and abbreviations. All drug names were extracted from the DRUG file of the FAERS and recorded. An archive of drug names that included the names of all preparations, generic names, and synonyms of drugs marketed worldwide was created using the Martindale website

(https://www.medicinescomplete.com/mc/login.ht $\mathrm{m})$. Phenytoin, carbamazepine, lamotrigine, topiramate, valproic acid, and ethotoin were identified by linking this archive with the FAERS database. All records that included AEDs in the DRUG files were selected, and the relevant reactions from the REACTION files were then identified.

Adverse events in the FAERS database were coded using the MedDRA ${ }^{\circledR}$ PTs, which are grouped by defined medical condition or area of interest. We identified PTs related to cancer using the Standardized MedDRA ${ }^{\circledR}$ Queries (SMQ). PTs related to 10 cancers (bladder cancer, colorectal cancer, lung cancer, pancreatic cancer, gastric cancer, esophageal cancer, hematological malignancies, melanoma, breast cancer, and prostate cancer) were identified in the SMQ category of malignant tumors.

\section{Data mining (disproportionality analysis)}

The reporting odds ratio (ROR) and information component (IC) were utilized to detect spontaneous report signals. Signal scores were calculated using a case/non-case method [15, 16]. ROR and IC are widely used algorithms that have been employed by the Netherlands Pharmacovigilance Centre and the World Health Organization, respectively [17, 18]. Those reports containing the event of interest were 
defined as the cases; all other reports comprised the non-cases.

Applying these algorithms and using a two-by-two table of frequency counts, we calculated signal scores to assess whether or not a drug was significantly associated with cancer diagnosis. However, these calculations or algorithms, so-called disproportionality analyses, differ from one another in that the ROR is frequentist (non-Bayesian)[17], whereas the IC is Bayesian[18]. For the ROR, an inverse signal was defined if the upper limit of the $95 \%$ two-sided confidence interval $(95 \% \mathrm{CI})$ was $<1$. For the IC, an inverse signal was defined if the upper limit of the $95 \%$ $\mathrm{CI}$ was $<0$. In the current study, two methods were used to detect inverse signals, and the association between AED and cancer was listed as an inverse signal when the two indices met the criteria outlined above. Data management and analyses were performed using Visual Mining Studio software (version 8.0; Mathematical Systems, Inc. Tokyo, Japan).

\section{Claims data}

\section{Data source}

A large and chronologically organized claims database constructed by the Japan Medical Data Center Co., Ltd (JMDC; Tokyo, Japan), using standardized disease classifications and anonymous record linkage, was employed in this study [19]. In total, this database includes approximately 3 million insured persons (approximately $2.5 \%$ of the population), comprised mainly of company employees and their family members. The JMDC claims database includes monthly claims from medical institutions and pharmacies submitted from January 2005 to May 2014. The database provides information on the beneficiaries, including encrypted personal identifiers, age, sex, International Classification of Diseases $10^{\text {th }}$ revision (ICD-10) procedure and diagnostic codes, as well as the name, dose, and number of days supplied of the prescribed and/or dispensed drugs. All drugs were coded according to the Anatomical Therapeutic Chemical classification of the European Pharmaceutical Market Research Association. An encrypted personal identifier was used to link claims data from different hospitals, clinics, and pharmacies. For the event sequence symmetry analysis (ESSA), we utilized cases extracted from the JMDC claims database for whom sodium channel-blocking AEDs were prescribed at least once during the study period and for whom a diagnosis of cancer was made.

This study was approved by the Ethics Committee of Kinki University School of Pharmacy. All personal data (name and identification number) from the JMDC claims database were replaced by a univocal numerical code, rendering the database anony- mous at the source. Therefore, there was no need to obtain informed consent in this study.

\section{Definition of AEDs and cancers}

Six sodium channel-blocking AEDs (phenytoin, carbamazepine, lamotorigine, topiramate, valproic acid, and ethotoin) were analyzed. The ICD-10 codes of C18 (malignant neoplasm of colon), C19 (malignant neoplasm of rectosigmoid junction) and C20 (malignant neoplasm of rectum) were selected as those defining colorectal cancer. In addition, the ICD-10 codes of C67 (malignant neoplasm of bladder), C34 (malignant neoplasm of bronchus and lung), C25 (malignant neoplasm of pancreas), C16 (malignant neoplasm of stomach), C15 (malignant neoplasm of esophagus), C81-96 (malignant neoplasms, stated or presumed to be primary, of lymphoid, hematopoietic and related tissue), C43 (malignant melanoma of skin), C50 (malignant neoplasm of breast), and C61 (malignant neoplasm of prostate) were selected as those defining bladder cancer, lung cancer, pancreatic cancer, gastric cancer, esophageal cancer, hematological malignancies, melanoma, breast cancer, and prostate cancer, respectively.

\section{Data mining (symmetry analysis)}

ESSA was performed to evaluate whether sodium channel-blocking AEDs decrease the risk of cancer. The ESSA method has been described in detail in several published studies investigating the associations between the use of certain targeted drugs and potential adverse events [20,21]. Briefly, the ESSA evaluates asymmetry in the distribution of an incident event before and after the initiation of a specific treatment. Asymmetry may indicate an association between the specific treatment of interest and the event. In this study, the inverse association between sodium channel-blocking AED use and the diagnosis of cancer was analyzed.

The crude sequence ratio (SR) is defined as the ratio of the number of patients newly diagnosed with cancer after relative to before the initiation of sodium channel-blocking AEDs. ASR $<1$ signified an inverse association of sodium channel-blocking AED use with a risk of cancer. The SR is sensitive to prescribing or event trends over time. Therefore, the SRs were adjusted for temporal trends in sodium channel-blocking AEDs and events, using the method proposed by Hallas [20]. The probability that sodium channel-blocking AEDs were prescribed first, in the absence of any causal relationship, can be estimated by a so-called null-effect SR [20]. The null-effect SR generated by the proposed model may be interpreted as a reference value for the SR. Therefore, the null-effect SR is the expected SR in the absence of any 
causal association, after accounting for incidence trends. By dividing the crude SR by the null-effect SR, an adjusted SR (ASR) corrected for temporal trends is obtained. A slightly modified model was used to account for the limited time interval allowed between sodium channel-blocking AED use and cancer diagnosis [21].

All incident users of sodium channel-blocking AEDs and all newly diagnosed cancer cases were identified from January 2005 to May 2014. Those patients were followed up until May 2014; therefore, different patients were followed-up over different periods. Incidence was defined as the first prescription of sodium channel-blocking AEDs. To exclude prevalent users of sodium channel-blocking AEDs, the analysis was restricted to users whose first prescription was administered in July 2005 or later (after a run-in period of 6 months). Likewise, the analysis was restricted to cases whose first diagnosis was in July 2005 or later. To ensure that our analysis was restricted to incident users of sodium channel-blocking AEDs and cases newly diagnosed with cancer, we also performed a waiting time distribution analysis [22]. An identical run-in period was also applied to patients enrolled in the cohort after June 2005. Incident users were identified by excluding those patients who received their first prescription for sodium channel-blocking AEDs before July 2005, and cases newly diagnosed with cancer were identified by excluding those patients whose first diagnosis of cancer was before July 2005. Those patients who had initiated a new treatment with sodium channel-blocking AEDs and whose first diagnosis of cancer was within a 36-month period of treatment initiation were identified. Patients who had received their first prescription for sodium channel-blocking AEDs and whose first diagnosis of cancer was within the same month were not included in determination of the SR.

The results of the analyses are expressed as means \pm standard deviations (SD) for quantitative data and as frequencies (percentages) for categorical data. The 95\% CI for the ASR was calculated using a method for exact CIs for binomial distributions [23].

\section{Results}

\section{FAERS database}

A total of 5,174 PTs were found in reports on phenytoin, 6,353 for carbamazepine, 5,908 for lamotrigine, 5,544 for topiramate, 6,625 for valproic acid, and 79 for ethotoin. The total number of drug-reaction pairs for sodium channel-blocking AEDs was 694,785, including 98,049 for phenytoin, 126,868 for carbamazepine, 170,433 for lamotrigine, 112,454 for topiramate, 186,889 for valproic acid, and 92 for ethotoin. The number of drug-reaction pairs was 17,495 for bladder cancer, 32,240 for colorectal cancer, 75,759 for lung cancer, 20,801 for pancreatic cancer, 10,207 for gastric cancer, 5,792 for esophageal cancer, 147,183 for hematological malignancies, 15,447 for melanoma, 165,170 for breast cancer, and 27,026 for prostate cancer.

The statistical data on sodium channel-blocking AED-associated cancers are presented in Table 1. The signal scores of individual cancers showed an inverse association with sodium channel-blocking AEDs (Figure 1). In the analysis of individual sodium channel-blocking AEDs, significant inverse signals were found for bladder cancer with phenytoin, carbamazepine, lamotrigine, topiramate, and valproic acid, for colorectal cancer with carbamazepine, lamotrigine, topiramate, and valproic acid, for lung cancer with phenytoin, carbamazepine, lamotrigine, topiramate, and valproic acid, for pancreatic cancer with phenytoin, carbamazepine, lamotrigine, topiramate, and valproic acid, for gastric cancer with phenytoin, lamotrigine, topiramate, and valproic acid, for esophageal cancer with lamotrigine, for hematological malignancies with phenytoin, carbamazepine, lamotrigine, topiramate, and valproic acid, for melanoma with phenytoin, carbamazepine, lamotrigine, topiramate, and valproic acid, for breast cancer with phenytoin, carbamazepine, lamotrigine, topiramate, and valproic acid, and for prostate cancer with carbamazepine, lamotrigine, topiramate, and valproic acid. No significant positive associations were found in this analysis.

Table 1. The associations between sodium channel-blocking AEDs and various cancers in the FAERS

\begin{tabular}{|c|c|c|c|c|c|c|}
\hline & Case & Non-cases & ROR & $95 \% \mathrm{CI}$ & IC & $95 \% \mathrm{CI}$ \\
\hline \multicolumn{7}{|l|}{ Bladder cancer } \\
\hline Sodium channel-blocking AEDs & 28 & 694,757 & $0.15^{*}$ & $0.10-0.22$ & $-2.69^{*}$ & -3.23 to -2.16 \\
\hline Phenytoin & 2 & 98,047 & $0.08^{*}$ & $0.02-0.30$ & $-3.19^{*}$ & -4.85 to -1.52 \\
\hline Carbamazepine & 7 & 126,861 & $0.21^{*}$ & $0.10-0.43$ & $-2.13^{*}$ & -3.15 to -1.11 \\
\hline Lamotrigine & 4 & 170,429 & $0.09^{*}$ & $0.03-0.23$ & $-3.23^{*}$ & -4.52 to -1.94 \\
\hline Topiramate & 6 & 112,448 & $0.20^{*}$ & $0.09-0.44$ & $-2.16^{*}$ & -3.25 to -1.07 \\
\hline Valproic acid & 9 & 186,880 & $0.18^{*}$ & $0.09-0.34$ & $-2.36^{*}$ & -3.27 to -1.44 \\
\hline Ethotoin & 0 & 92 & 0.00 & - & -0.04 & $-2.94-2.87$ \\
\hline \multicolumn{7}{|l|}{ Colorectal cancer } \\
\hline Sodium channel-blocking AEDs & 115 & 694,670 & $0.33^{*}$ & $0.28-0.40$ & $-1.57^{*}$ & -1.84 to -1.30 \\
\hline Phenytoin & 35 & 98,014 & 0.72 & $0.52-1.00$ & -0.46 & $-0.94-0.02$ \\
\hline Carbamazepine & 38 & 126,830 & $0.60^{*}$ & $0.44-0.83$ & $-0.71^{*}$ & -1.17 to -0.25 \\
\hline
\end{tabular}




\begin{tabular}{|c|c|c|c|c|c|c|}
\hline Lamotrigine & 8 & 170,425 & $0.09^{*}$ & $0.05-0.19$ & $-3.25^{*}$ & -4.21 to -2.28 \\
\hline Topiramate & 8 & 112,446 & $0.14^{*}$ & $0.07-0.29$ & $-2.65^{*}$ & -3.62 to -1.69 \\
\hline Valproic acid & 26 & 186,863 & $0.28^{*}$ & $0.19-0.41$ & $-1.79^{*}$ & -2.35 to -1.24 \\
\hline Ethotoin & 0 & 92 & 0.00 & - & -0.06 & $-2.97-2.84$ \\
\hline \multicolumn{7}{|l|}{ Lung cancer } \\
\hline Sodium channel-blocking AEDs & 284 & 694,501 & $0.35^{*}$ & $0.31-0.39$ & $-1.51^{*}$ & -1.68 to -1.33 \\
\hline Phenytoin & 72 & 97,977 & $0.63^{*}$ & $0.50-0.79$ & $-0.66^{*}$ & -0.99 to -0.32 \\
\hline Carbamazepine & 59 & 126,809 & $0.40^{*}$ & $0.31-0.52$ & $-1.31^{*}$ & -1.68 to -0.94 \\
\hline Lamotrigine & 35 & 170,398 & $0.18^{*}$ & $0.13-0.25$ & $-2.47^{*}$ & -2.95 to -1.99 \\
\hline Topiramate & 25 & 112,429 & $0.19^{*}$ & $0.13-0.28$ & $-2.34^{*}$ & -2.91 to -1.78 \\
\hline Valproic acid & 93 & 186,796 & $0.43^{*}$ & $0.35-0.52$ & $-1.22^{*}$ & -1.51 to -0.92 \\
\hline Ethotoin & 0 & 92 & 0.00 & - & -0.15 & $-3.05-2.75$ \\
\hline \multicolumn{7}{|l|}{ Pancreatic cancer } \\
\hline Sodium channel-blocking AEDs & 57 & 694,728 & $0.25^{*}$ & $0.20-0.33$ & $-1.94^{*}$ & -2.32 to -1.56 \\
\hline Phenytoin & 9 & 98,040 & $0.29^{*}$ & $0.15-0.55$ & $-1.69^{*}$ & -2.60 to -0.78 \\
\hline Carbamazepine & 10 & 126,858 & $0.25^{*}$ & $0.13-0.46$ & $-1.92^{*}$ & -2.79 to -1.05 \\
\hline Lamotrigine & 6 & 170,427 & $0.11^{*}$ & $0.05-0.24$ & $-2.99^{*}$ & -4.08 to -1.89 \\
\hline Topiramate & 11 & 112,443 & $0.31^{*}$ & $0.17-0.55$ & $-1.62^{*}$ & -2.45 to -0.79 \\
\hline Valproic acid & 21 & 186,868 & $0.35^{*}$ & $0.23-0.54$ & $-1.46^{*}$ & -2.08 to -0.85 \\
\hline Ethotoin & 0 & 92 & 0.00 & - & -0.04 & $-2.94-2.86$ \\
\hline \multicolumn{7}{|l|}{ Gastric cancer } \\
\hline Sodium channel-blocking AEDs & 35 & 694,750 & $0.32^{*}$ & $0.23-0.44$ & $-1.61^{*}$ & -2.09 to -1.13 \\
\hline Phenytoin & 5 & 98,044 & $0.33^{*}$ & $0.14-0.78$ & $-1.45^{*}$ & -2.63 to -0.27 \\
\hline Carbamazepine & 18 & 126,850 & 0.91 & $0.57-1.44$ & -0.14 & $-0.80-0.53$ \\
\hline Lamotrigine & 2 & 170,431 & $0.07^{*}$ & $0.02-0.30$ & $-3.21^{*}$ & -4.87 to -1.54 \\
\hline Topiramate & 5 & 112,449 & $0.28^{*}$ & $0.12-0.68$ & $-1.63^{*}$ & -2.81 to -0.46 \\
\hline Valproic acid & 5 & 186,884 & $0.17^{*}$ & $0.07-0.41$ & $-2.34^{*}$ & -3.51 to -1.16 \\
\hline Ethotoin & 0 & 92 & 0.00 & - & -0.02 & $-2.92-2.88$ \\
\hline \multicolumn{7}{|l|}{ Esophageal cancer } \\
\hline Sodium channel-blocking AEDs & 30 & 694,755 & $0.48^{*}$ & $0.34-0.69$ & $-1.02^{*}$ & -1.54 to -0.50 \\
\hline Phenytoin & 5 & 98,044 & 0.57 & $0.24-1.38$ & -0.70 & $-1.87-0.48$ \\
\hline Carbamazepine & 6 & 126,862 & 0.53 & $0.24-1.18$ & -0.81 & $-1.90-0.28$ \\
\hline Lamotrigine & 6 & 170,427 & $0.40^{*}$ & $0.18-0.88$ & $-1.21^{*}$ & -2.30 to -0.12 \\
\hline Topiramate & 0 & 112,454 & 0.00 & - & $-3.46^{*}$ & -6.35 to -0.57 \\
\hline Valproic acid & 12 & 186,877 & 0.72 & $0.41-1.27$ & -0.44 & $-1.24-0.36$ \\
\hline Ethotoin & 0 & 92 & 0.00 & - & -0.01 & $-2.91-2.89$ \\
\hline \multicolumn{7}{|l|}{ Hematological malignancies } \\
\hline Sodium channel-blocking AEDs & 508 & 694,277 & $0.32^{*}$ & $0.29-0.35$ & $-1.63^{*}$ & -1.75 to -1.50 \\
\hline Phenytoin & 115 & 97,934 & $0.52^{*}$ & $0.43-0.62$ & $-0.94^{*}$ & -1.21 to -0.67 \\
\hline Carbamazepine & 121 & 126,747 & $0.42^{*}$ & $0.35-0.50$ & $-1.24^{*}$ & -1.50 to -0.98 \\
\hline Lamotrigine & 62 & 170,371 & $0.16^{*}$ & $0.13-0.21$ & $-2.62^{*}$ & -2.98 to -2.25 \\
\hline Topiramate & 33 & 112,421 & $0.13^{*}$ & $0.09-0.18$ & $-2.91^{*}$ & -3.40 to -2.41 \\
\hline Valproic acid & 177 & 186,712 & $0.42^{*}$ & $0.36-0.48$ & $-1.25^{*}$ & -1.47 to -1.03 \\
\hline Ethotoin & 0 & 92 & 0.00 & - & -0.28 & $-3.18-2.63$ \\
\hline \multicolumn{7}{|l|}{ Melanoma } \\
\hline Sodium channel-blocking AEDs & 63 & 694,722 & $0.38^{*}$ & $0.30-0.49$ & $-1.37^{*}$ & -1.73 to -1.01 \\
\hline Phenytoin & 9 & 98,040 & $0.39^{*}$ & $0.20-0.74$ & $-1.28^{*}$ & -2.19 to -0.37 \\
\hline Carbamazepine & 9 & 126,859 & $0.30^{*}$ & $0.16-0.57$ & $-1.64^{*}$ & -2.55 to -0.72 \\
\hline Lamotrigine & 16 & 170,417 & $0.40^{*}$ & $0.24-0.65$ & $-1.28^{*}$ & -1.98 to -0.58 \\
\hline Topiramate & 11 & 112,443 & $0.41^{*}$ & $0.23-0.74$ & $-1.21^{*}$ & -2.04 to -0.37 \\
\hline Valproic acid & 18 & 186,871 & $0.41^{*}$ & $0.26-0.64$ & $-1.25^{*}$ & -1.92 to -0.59 \\
\hline Ethotoin & 0 & 92 & 0.00 & - & -0.03 & $-2.93-2.87$ \\
\hline \multicolumn{7}{|l|}{ Breast cancer (female) } \\
\hline Sodium channel-blocking AEDs & 372 & 694,413 & $0.21^{*}$ & $0.19-0.23$ & $-2.24^{*}$ & -2.39 to -2.09 \\
\hline Phenytoin & 61 & 97,988 & $0.24^{*}$ & $0.19-0.31$ & $-2.01^{*}$ & -2.38 to -1.64 \\
\hline Carbamazepine & 61 & 126,807 & $0.19^{*}$ & $0.15-0.24$ & $-2.38^{*}$ & -2.75 to -2.01 \\
\hline Lamotrigine & 88 & 170,345 & $0.20^{*}$ & $0.16-0.25$ & $-2.28^{*}$ & -2.59 to -1.98 \\
\hline Topiramate & 77 & 112,377 & $0.27^{*}$ & $0.22-0.34$ & $-1.88^{*}$ & -2.20 to -1.55 \\
\hline Valproic acid & 85 & 186,804 & $0.18^{*}$ & $0.14-0.22$ & $-2.47^{*}$ & -2.78 to -2.15 \\
\hline Ethotoin & 0 & 92 & 0.00 & - & -0.31 & $-3.21-2.60$ \\
\hline \multicolumn{7}{|l|}{ Prostate cancer (male) } \\
\hline Sodium channel-blocking AEDs & 78 & 694,707 & $0.27^{*}$ & $0.21-0.34$ & $-1.87^{*}$ & -2.20 to -1.55 \\
\hline Phenytoin & 31 & 98,018 & 0.76 & $0.54-1.08$ & -0.38 & $-0.89-0.13$ \\
\hline Carbamazepine & 17 & 126,851 & $0.32^{*}$ & $0.20-0.52$ & $-1.58^{*}$ & -2.26 to -0.89 \\
\hline Lamotrigine & 13 & 170,420 & $0.18^{*}$ & $0.11-0.32$ & $-2.36^{*}$ & -3.13 to -1.59 \\
\hline Topiramate & 7 & 112,447 & $0.15^{*}$ & $0.07-0.31$ & $-2.57^{*}$ & -3.59 to -1.55 \\
\hline Valproic acid & 10 & 186,879 & $0.13^{*}$ & $0.07-0.24$ & $-2.84^{*}$ & -3.71 to -1.97 \\
\hline Ethotoin & 0 & 92 & 0.00 & - & -0.05 & $-2.96-2.85$ \\
\hline
\end{tabular}

AED: Antiepileptic drug . FAERS: The US Food and Drug Administration (FDA) Adverse Event Reporting System. Case: Number of reports of cancer. Non-cases: Number of reports of adverse drug reactions other than cancer. ROR: Reporting odds ratio. CI: Confidence interval. IC: Information component. *: Significant 


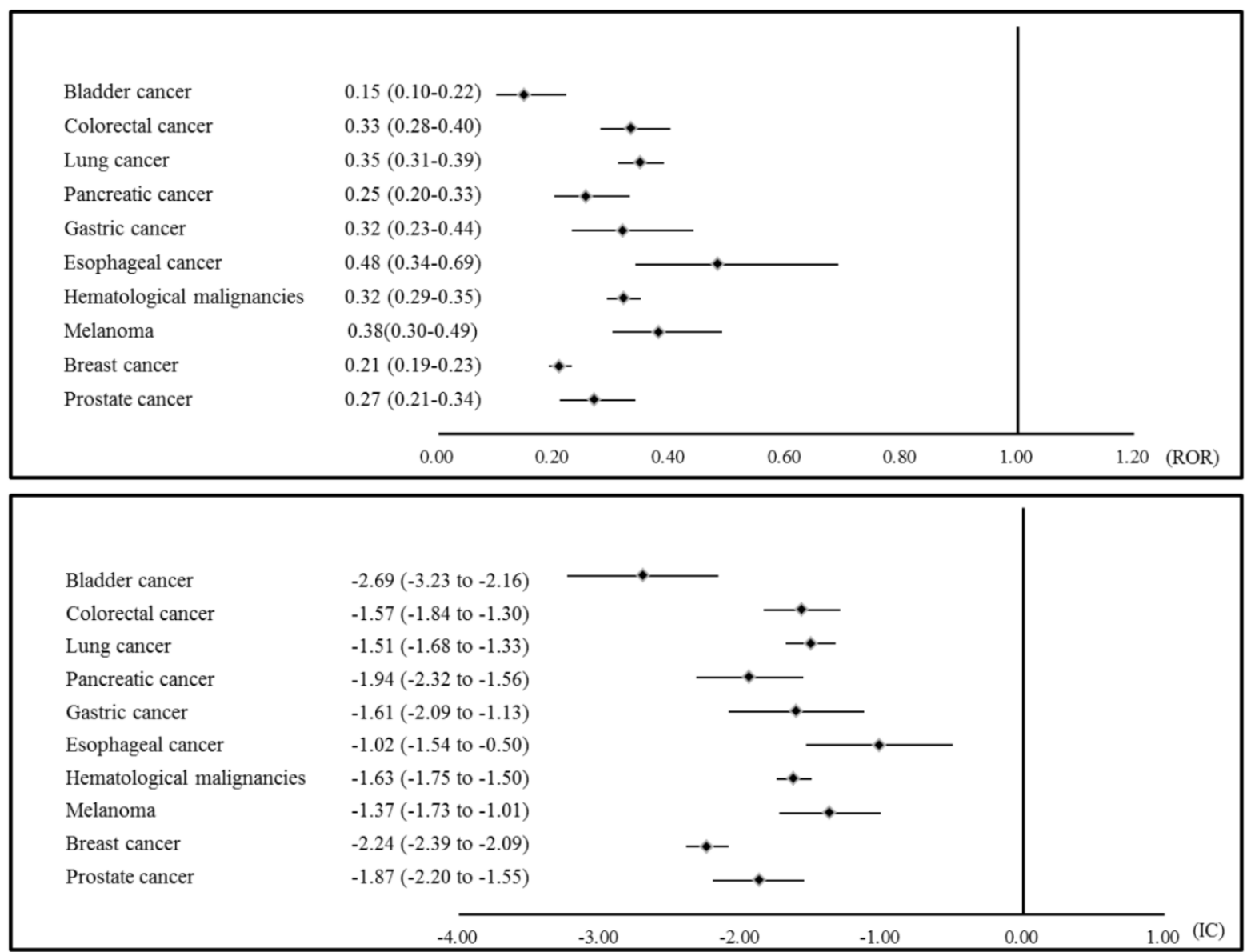

Figure 1. Disproportionality analysis: the association between sodium channel-blocking AEDs and cancers. AED: Antiepileptic drug; ROR: Reporting odds ratio; IC: Information component.

Table 2. Characteristics of the study population for sodium channel-blocking AED users (January 2005 to May 2014)

\begin{tabular}{llll}
\hline & Total & Males & Females \\
\hline Users, $\mathrm{n}$ & 34,473 & & \\
$\begin{array}{l}\text { Claims including AEDs, } \mathrm{n} \\
\text { Incident users, } \mathrm{n}(\%)\end{array}$ & 729,441 & & \\
Age, years, $\mathrm{n}(\%)$ & 17,610 & $8,490(48.2)$ & $9,120(51.8)$ \\
$<20$ & & & \\
$20-39$ & $3,332(18.9)$ & $1,665(19.6)$ & $1,667(18.3)$ \\
$40-59$ & $6,848(38.9)$ & $3,096(36.5)$ & $3,752(41.1)$ \\
$60-79$ & $6,226(35.4)$ & $3,171(37.3)$ & $3,055(33.5)$ \\
$\geqq 80$ & $1,204(6.8)$ & $558(6.6)$ & $646(7.1)$ \\
Mean \pm SD & 0 & 0 & 0 \\
\hline $\begin{array}{l}\text { Incident users: Number of patients who received their first prescription for sodium } \\
\text { channel-blocking AEDs } \\
\text { AED: Antiepileptic drug }\end{array}$ & & \\
SD: Standard deviation & $35.1 \pm 16.7$ & $35.3 \pm 16.8$ & $35.0 \pm 16.5$ \\
\end{tabular}

\section{JMDC claims database}

The ESSA characteristics of the study population are summarized in Table 2. The number of claims pertaining to sodium channel-blocking AEDs during the study period was 729,441 . Among 34,473 sodium channel-blocking AED users, 17,610 incident users were identified, the mean age of whom was $35.1 \pm 16.7$ years. Table 3 shows the associations between sodium channel-blocking AED use and the risk of cancers. Of the 17,610 incident sodium channel-blocking AED users, there were 158 with a diagnosis of bladder cancer, 647 with colorectal cancer, 408 with lung cancer, 265 with pancreatic cancer, 487 with gastric cancer, 40 with esophageal cancer, 299 with hematological malignancies, and 20 with melanoma. Of the 9,120 female and 8,490 male incident sodium channel-blocking AED users, 262 and 146 had a diagnosis of breast cancer and prostate cancer, respectively, before or after the initiation of sodium channel-blocking AEDs. Sodium channel-blocking AED use was inversely associated with diagnoses of colorectal cancer, lung cancer, gastric cancer, and hematological malignancies, with ASRs of 0.72 (95\% CI: $0.60-0.86), 0.65(0.51-0.81), 0.80(0.65-0.98)$, and $0.50(0.37-0.66)$, respectively (Figure 2). Analyses of bladder cancer, pancreatic cancer, esophageal cancer, melanoma, breast cancer, and prostate cancer diagnoses showed no significant inverse associations with sodium channel-blocking AED use. In the analyses of individual sodium channel-blocking AEDs, phenytoin was inversely associated with diagnoses of colorectal cancer (ASR: 0.53, 95\% CI: 0.33 - 0.83), lung cancer 
$(0.30,0.17-0.50)$, gastric cancer $(0.49,0.29-0.83)$, hematological malignancies $(0.25,0.13-0.47)$, and breast cancer $(0.35,0.12-0.86)$. Carbamazepine was inversely associated with a diagnosis of colorectal cancer $(0.74,0.56-0.97)$. Valproic acid was inversely associated with diagnoses of colorectal cancer $(0.63$, $0.49-0.81)$, lung cancer $(0.60,0.44-0.82)$, and hematological malignancies $(0.45,0.30-0.66)$. No signifi-

A) Colorectal cancer

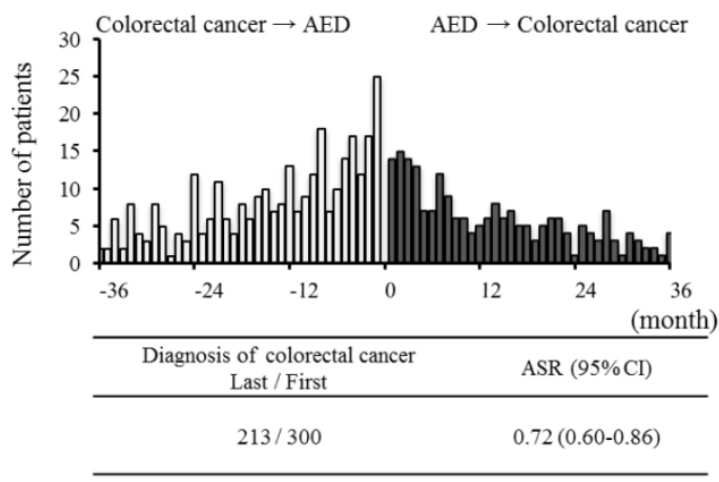

C) Gastric cancer

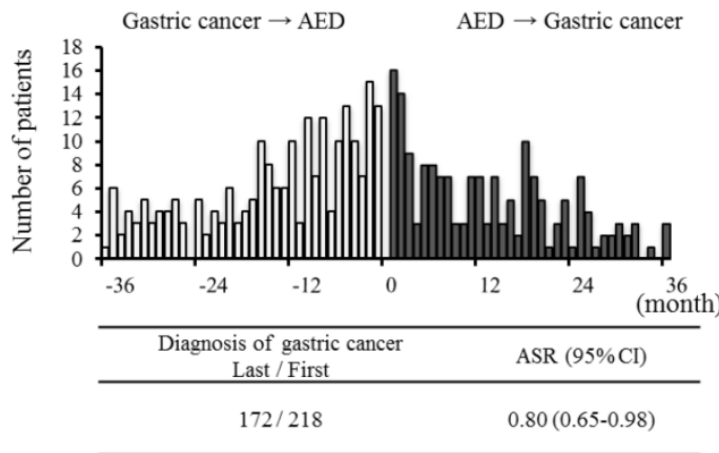

cant inverse associations were found in the analyses of lamotrigine, topiramate, and ethotoin. No significant positive associations were found in this analysis.

A summary of the inverse signals detected for sodium channel-blocking AED-associated cancers is presented in Table 4.

B) Lung cancer

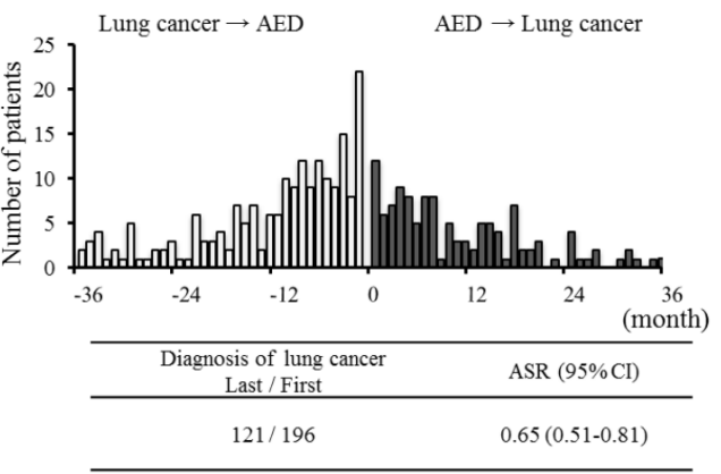

D) Hematological malignancies

Hematological malignancies $\rightarrow$ AED $\quad$ AED $\rightarrow$ Hematological malignancies

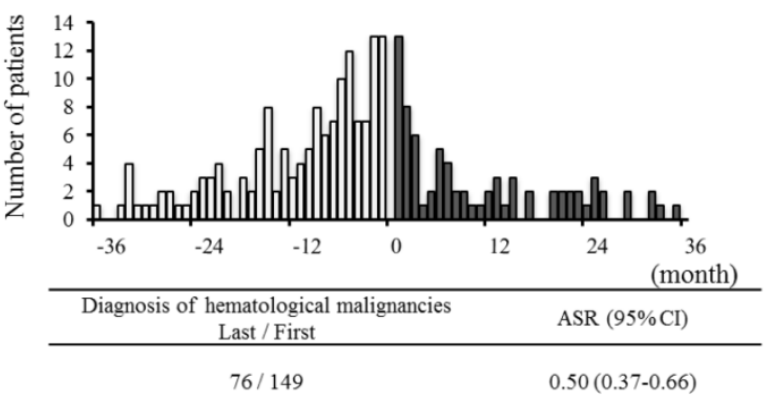

Figure 2. Event sequence symmetry analysis: the association between sodium channel-blocking antiepileptic drugs and cancers. AED: Antiepileptic drug; ASR: Adjusted sequence ratio; $\mathrm{Cl}$ : Confidence interval.

Table 3. Symmetry analysis: the associations between sodium channel-blocking AEDs and cancers

\begin{tabular}{|c|c|c|c|c|c|c|c|}
\hline & \multirow{2}{*}{$\begin{array}{l}\text { Incident } \\
\text { users }\end{array}$} & \multirow{2}{*}{$\begin{array}{l}\text { Cases with } \\
\text { cancer }\end{array}$} & \multirow{2}{*}{\multicolumn{2}{|c|}{$\begin{array}{l}\text { Diagnosis of cancer } \\
\text { last/first }\end{array}$}} & \multirow[t]{2}{*}{ Adjusted SR } & \multicolumn{2}{|l|}{$95 \% \mathrm{CI}$} \\
\hline & & & & & & Lower & Upper \\
\hline \multicolumn{8}{|l|}{ Bladder cancer } \\
\hline Sodium channel-blocking AEDs & 17,610 & 158 & 61 & 66 & 0.89 & 0.61 & 1.27 \\
\hline Phenytoin & 2,008 & 28 & 11 & 13 & 0.72 & 0.29 & 1.74 \\
\hline Carbamazepine & 5,956 & 70 & 25 & 26 & 0.89 & 0.50 & 1.61 \\
\hline Lamotrigine & 2,473 & 11 & 5 & 5 & 1.23 & 0.28 & 5.34 \\
\hline Topiramate & 617 & 2 & 1 & 1 & 0.99 & 0.01 & 77.71 \\
\hline Valproic acid & 10,961 & 81 & 32 & 35 & 0.87 & 0.52 & 1.45 \\
\hline Ethotoin & 1 & 0 & 0 & 0 & - & - & - \\
\hline \multicolumn{8}{|l|}{ Colorectal cancer } \\
\hline Sodium channel-blocking AEDs & 17,610 & 647 & 213 & 300 & $0.72^{*}$ & 0.60 & 0.86 \\
\hline Phenytoin & 2,008 & 105 & 32 & 54 & $0.53^{*}$ & 0.33 & 0.83 \\
\hline Carbamazepine & 5,956 & 279 & 97 & 127 & $0.74^{*}$ & 0.56 & 0.97 \\
\hline Lamotrigine & 2,473 & 64 & 22 & 31 & 0.94 & 0.52 & 1.67 \\
\hline Topiramate & 617 & 20 & 6 & 8 & 0.78 & 0.22 & 2.55 \\
\hline Valproic acid & 10,961 & 358 & 106 & 168 & $0.63^{*}$ & 0.49 & 0.81 \\
\hline Ethotoin & 1 & 0 & 0 & 0 & - & - & - \\
\hline \multicolumn{8}{|l|}{ Lung cancer } \\
\hline Sodium channel-blocking AEDs & 17,610 & 408 & 121 & 196 & $0.65^{*}$ & 0.51 & 0.81 \\
\hline
\end{tabular}




\begin{tabular}{|c|c|c|c|c|c|c|c|}
\hline \multirow[b]{3}{*}{ Phenytoin } & \multirow{3}{*}{$\begin{array}{l}\text { Incident } \\
\text { users }\end{array}$} & \multirow{3}{*}{$\begin{array}{l}\text { Cases with } \\
\text { cancer }\end{array}$} & \multirow{2}{*}{\multicolumn{2}{|c|}{$\begin{array}{l}\text { Diagnosis of cancer } \\
\text { last/first }\end{array}$}} & \multirow{3}{*}{$\begin{array}{l}\text { Adjusted SR } \\
0.30^{*}\end{array}$} & \multicolumn{2}{|l|}{$95 \% \mathrm{CI}$} \\
\hline & & & & & & \multirow{2}{*}{$\begin{array}{l}\text { Lower } \\
0.17\end{array}$} & \multirow{2}{*}{$\begin{array}{l}\text { Upper } \\
0.50\end{array}$} \\
\hline & & & 20 & 62 & & & \\
\hline Carbamazepine & 5,956 & 146 & 51 & 63 & 0.81 & 0.55 & 1.19 \\
\hline Lamotrigine & 2,473 & 25 & 10 & 11 & 1.27 & 0.48 & 3.30 \\
\hline Topiramate & 617 & 12 & 3 & 6 & 0.54 & 0.09 & 2.52 \\
\hline Valproic acid & 10,961 & 224 & 65 & 113 & $0.60^{*}$ & 0.44 & 0.82 \\
\hline Ethotoin & 1 & 0 & 0 & 0 & - & - & - \\
\hline \multicolumn{8}{|l|}{ Pancreatic cancer } \\
\hline Sodium channel-blocking AEDs & 17,610 & 265 & 102 & 109 & 0.93 & 0.70 & 1.22 \\
\hline Phenytoin & 2,008 & 52 & 17 & 24 & 0.62 & 0.31 & 1.20 \\
\hline Carbamazepine & 5,956 & 112 & 46 & 40 & 1.09 & 0.70 & 1.72 \\
\hline Lamotrigine & 2,473 & 20 & 7 & 10 & 0.91 & 0.30 & 2.66 \\
\hline Topiramate & 617 & 8 & 0 & 4 & - & - & - \\
\hline Valproic acid & 10,961 & 136 & 55 & 61 & 0.89 & 0.61 & 1.30 \\
\hline Ethotoin & 1 & 0 & 0 & 0 & - & - & - \\
\hline \multicolumn{8}{|l|}{ Gastric cancer } \\
\hline Sodium channel-blocking AEDs & 17,610 & 487 & 172 & 218 & $0.80^{*}$ & 0.65 & 0.98 \\
\hline Phenytoin & 2,008 & 88 & 25 & 45 & $0.49^{*}$ & 0.29 & 0.83 \\
\hline Carbamazepine & 5,956 & 202 & 75 & 86 & 0.85 & 0.61 & 1.17 \\
\hline Lamotrigine & 2,473 & 51 & 15 & 29 & 0.69 & 0.35 & 1.34 \\
\hline Topiramate & 617 & 16 & 4 & 9 & 0.46 & 0.10 & 1.65 \\
\hline Valproic acid & 10,961 & 263 & 89 & 119 & 0.75 & 0.57 & 1.00 \\
\hline Ethotoin & 1 & 0 & 0 & 0 & - & - & - \\
\hline \multicolumn{8}{|l|}{ Esophageal cancer } \\
\hline Sodium channel-blocking AEDs & 17,610 & 40 & 14 & 20 & 0.64 & 0.30 & 1.33 \\
\hline Phenytoin & 2,008 & 4 & 1 & 3 & 0.27 & 0.01 & 3.34 \\
\hline Carbamazepine & 5,956 & 21 & 8 & 11 & 0.64 & 0.23 & 1.76 \\
\hline Lamotrigine & 2,473 & 10 & 6 & 3 & 2.35 & 0.50 & 14.53 \\
\hline Topiramate & 617 & 3 & 1 & 2 & 0.47 & 0.01 & 9.02 \\
\hline Valproic acid & 10,961 & 21 & 6 & 11 & 0.50 & 0.15 & 1.46 \\
\hline Ethotoin & 1 & 0 & 0 & 0 & - & - & - \\
\hline \multicolumn{8}{|l|}{ Hematological malignancies } \\
\hline Sodium channel-blocking AEDs & 17,610 & 299 & 76 & 149 & $0.50^{*}$ & 0.37 & 0.66 \\
\hline Phenytoin & 2,008 & 85 & 14 & 48 & $0.25^{*}$ & 0.13 & 0.47 \\
\hline Carbamazepine & 5,956 & 105 & 34 & 42 & 0.77 & 0.47 & 1.23 \\
\hline Lamotrigine & 2,473 & 24 & 12 & 10 & 1.54 & 0.61 & 3.98 \\
\hline Topiramate & 617 & 3 & 1 & 1 & 1.01 & 0.01 & 79.17 \\
\hline Valproic acid & 10,961 & 164 & 39 & 85 & $0.45^{*}$ & 0.30 & 0.66 \\
\hline Ethotoin & 1 & 0 & 0 & 0 & - & - & - \\
\hline \multicolumn{8}{|l|}{ Melanoma } \\
\hline Sodium channel-blocking AEDs & 17,610 & 20 & 10 & 6 & 1.43 & 0.47 & 4.78 \\
\hline Phenytoin & 2,008 & 3 & 3 & 0 & - & - & - \\
\hline Carbamazepine & 5,956 & 5 & 1 & 3 & 0.28 & 0.01 & 3.46 \\
\hline Lamotrigine & 2,473 & 4 & 1 & 3 & 0.37 & 0.01 & 4.55 \\
\hline Topiramate & 617 & 1 & 1 & 0 & - & - & - \\
\hline Valproic acid & 10,961 & 11 & 6 & 2 & 2.56 & 0.46 & 25.90 \\
\hline Ethotoin & 1 & 0 & 0 & 0 & - & - & - \\
\hline Breast cancer (female) & & & & & & & \\
\hline Sodium channel-blocking AEDs & 9,120 & 262 & 97 & 112 & 0.93 & 0.70 & 1.23 \\
\hline Phenytoin & 2,008 & 36 & 7 & 19 & $0.35^{*}$ & 0.12 & 0.86 \\
\hline Carbamazepine & 5,956 & 104 & 43 & 42 & 1.05 & 0.67 & 1.64 \\
\hline Lamotrigine & 2,473 & 36 & 16 & 16 & 1.47 & 0.69 & 3.14 \\
\hline Topiramate & 617 & 16 & 6 & 7 & 0.94 & 0.26 & 3.26 \\
\hline Valproic acid & 10,961 & 148 & 53 & 63 & 0.90 & 0.61 & 1.32 \\
\hline Ethotoin & 1 & 0 & 0 & 0 & - & - & - \\
\hline Prostate cancer (male) & & & & & & & \\
\hline Sodium channel-blocking AEDs & 8,490 & 146 & 51 & 65 & 0.85 & 0.58 & 1.24 \\
\hline Phenytoin & 2,008 & 38 & 16 & 16 & 0.95 & 0.45 & 2.03 \\
\hline Carbamazepine & 5,956 & 57 & 14 & 26 & 0.56 & 0.27 & 1.11 \\
\hline Lamotrigine & 2,473 & 18 & 6 & 9 & 0.98 & 0.29 & 3.09 \\
\hline Topiramate & 617 & 6 & 1 & 3 & 0.37 & 0.01 & 4.60 \\
\hline Valproic acid & 10,961 & 79 & 31 & 36 & 0.93 & 0.56 & 1.55 \\
\hline Ethotoin & 1 & 0 & 0 & 0 & - & - & - \\
\hline
\end{tabular}

AED: Antiepileptic drug. Adjusted SR: Adjusted sequence ratio. CI: Confidence interval.

All patients who initiated new treatment with sodium channel-blocking AEDs and whose first diagnosis of cancer was within a 36-month period were identified. Incident users: Number of patients who received their first prescription for sodium channel-blocking AEDs

Cases with cancer: Number of patients newly diagnosed with cancer

Diagnosis of cancer last: Number of patients with a diagnosis made after sodium channel-blocking AED use

Diagnosis of cancer first: Number of patients with a diagnosis made before sodium channel-blocking AED use

*: Significant 
Table 4. Summary of the inverse signals detected for sodium channel-blocking AED-associated cancers

\begin{tabular}{|c|c|c|c|c|c|c|c|c|c|c|c|c|c|c|c|c|c|c|c|c|}
\hline & \multicolumn{2}{|c|}{ Bladder } & \multicolumn{2}{|c|}{ Colorectal } & \multicolumn{2}{|c|}{ Lung } & \multicolumn{2}{|c|}{ Pancreatic } & \multicolumn{2}{|c|}{ Gastric } & \multicolumn{2}{|c|}{ Esophageal } & \multicolumn{2}{|c|}{ Hematological } & \multicolumn{2}{|c|}{ Melanoma } & \multicolumn{2}{|c|}{ Breast } & \multicolumn{2}{|c|}{ Prostate } \\
\hline & F & $\mathrm{J}$ & $\mathrm{F}$ & $\mathrm{J}$ & $\mathrm{F}$ & $\mathrm{J}$ & $\mathrm{F}$ & $\mathrm{J}$ & $\mathrm{F}$ & $\mathrm{J}$ & $\mathrm{F}$ & $\mathrm{J}$ & $\mathrm{F}$ & $\mathrm{J}$ & $\mathrm{F}$ & $\mathrm{J}$ & $\mathrm{F}$ & $\mathrm{J}$ & $\mathrm{F}$ & $\mathrm{J}$ \\
\hline Sodium channel-blocking AEDs & $\downarrow$ & nd & $\downarrow$ & $\downarrow$ & $\downarrow$ & $\downarrow$ & $\downarrow$ & nd & $\downarrow$ & $\downarrow$ & $\downarrow$ & nd & $\downarrow$ & $\downarrow$ & $\downarrow$ & nd & $\downarrow$ & nd & $\downarrow$ & nd \\
\hline Phenytoin & $\downarrow$ & nd & nd & $\downarrow$ & $\downarrow$ & $\downarrow$ & $\downarrow$ & nd & $\downarrow$ & $\downarrow$ & nd & nd & $\downarrow$ & $\downarrow$ & $\downarrow$ & nd & $\downarrow$ & $\downarrow$ & nd & nd \\
\hline Carbamazepine & $\downarrow$ & nd & $\downarrow$ & $\downarrow$ & $\downarrow$ & nd & $\downarrow$ & nd & nd & nd & nd & nd & $\downarrow$ & nd & $\downarrow$ & nd & $\downarrow$ & nd & $\downarrow$ & nd \\
\hline Lamotrigine & $\downarrow$ & nd & $\downarrow$ & nd & $\downarrow$ & nd & $\downarrow$ & nd & $\downarrow$ & nd & $\downarrow$ & nd & $\downarrow$ & nd & $\downarrow$ & nd & $\downarrow$ & nd & $\downarrow$ & nd \\
\hline Topiramate & $\downarrow$ & nd & $\downarrow$ & nd & $\downarrow$ & nd & $\downarrow$ & nd & $\downarrow$ & nd & nd & nd & $\downarrow$ & nd & $\downarrow$ & nd & $\downarrow$ & nd & $\downarrow$ & nd \\
\hline Valproic acid & $\downarrow$ & nd & $\downarrow$ & $\downarrow$ & $\downarrow$ & $\downarrow$ & $\downarrow$ & nd & $\downarrow$ & nd & nd & nd & $\downarrow$ & $\downarrow$ & $\downarrow$ & nd & $\downarrow$ & nd & $\downarrow$ & nd \\
\hline Ethotoin & nd & nd & nd & nd & nd & nd & nd & nd & nd & nd & nd & nd & nd & nd & nd & nd & nd & nd & nd & nd \\
\hline
\end{tabular}

F: The US Food and Drug Administration (FDA) Adverse Event Reporting System (FAERS)

J: The Japan Medical Data Center (JMDC) claims database

nd: No signal was detected.

$\downarrow$ : A negative signal was detected.

AED: Antiepileptic drug

\section{Discussion}

In analyses of both the FAERS and JMDC claims databases, significant inverse signals for colorectal cancer, lung cancer, gastric cancer, and hematological malignancies were found for sodium channel-blocking AEDs as a class. Consistent findings from the independent analyses involving different methodologies, algorithms, and databases suggest that sodium channel-blocking AED use is inversely associated with the risks of these cancers. For bladder cancer, pancreatic cancer, esophageal cancer, melanoma, breast cancer, and prostate cancer, significant inverse associations with sodium channel-blocking AEDs as a class were found in the analysis of the FAERS database, but not the JMDC claims database. Therefore, we determined that sodium channel-blocking AEDs as a class have no inverse association with these cancers.

In the analyses of individual sodium channel-blocking AEDs, significant inverse associations were found for colorectal cancer with both carbamazepine and valproic acid, for lung cancer with both phenytoin and valproic acid, for gastric cancer with phenytoin, for hematological malignancies with both phenytoin and valproic acid, and for breast cancer with phenytoin. Of note, significant positive signals between sodium channel-blocking AEDs and cancer risk were not found in the analyses. Although there has been no definite clinical evidence at the present time, we have clearly showed an inverse association between sodium channel-blocking AEDs and several cancers by analyzing different databases using different methodologies. These consistent findings may suggest that sodium channel-blocking AEDs are associated with decreased risk of certain cancers.

Yang et al. reported that phenytoin suppresses $\mathrm{Na}^{+}$currents in VGSC-expressing metastatic breast cancer cells, thus blocking VGSC-dependent migration and invasion [13]. This experimental study has suggested that phenytoin may have potential chemopreventative effects against breast cancer. Recently,
Nelson et al. reported that treatment with phenytoin significantly reduced breast tumor growth and metastasis in vivo [24]. Although phenytoin is expected to be a potential anticancer drug candidate, there is no clinical evidence that phenytoin use is associated with a decreased risk of cancer. In our study, a significant inverse association between breast cancer and phenytoin was found in analyses of the FAERS and JMDC claims databases. This accumulation of evidence, including our study, supports the hypothesis that phenytoin use may be associated with a decreased risk of breast cancer. Additionally, some studies have suggested that phenytoin also inhibits migration and secretion in prostate cancer cells [10, 25]. In our study, an inverse association of phenytoin with prostate cancer risk was not found, but associations with lung cancer, gastric cancer, hematological malignancies, and breast cancer were detected in analyses of the FAERS and JMDC claims databases. These findings support the hypothesis that phenytoin may be a possible anticancer drug candidate.

Significant inverse associations were found for valproic acid with colorectal cancer, lung cancer, and hematological malignancies in analyses of both the FAERS and JMDC claims databases. Valproic acid is a VGSC-targeting AED, but several experimental and clinical studies have also been performed to evaluate the anticancer effects of valproic acid as a histone deacetylase (HDAC) inhibitor. Histone acetylation represents an epigenetic change and plays important roles in the initiation and progression of cancer [26, 27]. Meanwhile, deacetylation of histones induces transcriptional repression through chromatin condensation. HDACs play important roles in transcriptional regulation and pathogenesis of cancer and have also been shown to downregulate angiogenesis-related gene expression in endothelial and tumor cells $[28,29]$. HDAC inhibitors induce differentiation, cell growth arrest and apoptosis by promoting gene transcription in different cancer cell types [30,31]. Thus, HDAC inhibitors are considered to be potential drug candidates for differentiation therapy of cancer. 
A number of in vivo and in vitro studies demonstrated that VPA is a strong HDAC inhibitor and is effective for regulating the growth, differentiation, and apoptosis of cancer cells as well as for blocking angiogenesis [32-35]. To date, three HDAC inhibitors (vorinostat, romidepsin, and belinostat) have been approved by the FDA for the treatment of cutaneous T-cell lymphoma [36]. Currently, these three drugs are undergoing further evaluation in other diseases, including hematological malignancies and solid tumors, either as a single agent or in combination with other drugs [36].

Experimental and clinical investigations have investigated VPA as a potential anticancer drug candidate [37-39]. In addition, clinical studies have been designed to evaluate the efficacy and safety of combination therapies involving VPA and anticancer agents, including demethylating or hypomethylating agents, in patients with advanced-stage cancers [40-42]. Recently, a retrospective cohort study by Kang et al. showed that use of VPA is associated with a lower risk of developing head and neck cancer [43]. However, data from these studies are inadequate to determine whether the use of VPA reduces the risk of cancer. Although no definite clinical evidence exists at the present time, our study supports the hypothesis that valproic acid may be a potential anticancer drug candidate.

Few studies have addressed the potential anticancer effect of VPA as a sodium channel-blocking agent. However, the anticancer effects of VPA might be attributable to the effects of both HDAC inhibitor and sodium channel-blocking agent. Further studies are needed to evaluate this.

To date, there have been no reports of an association between carbamazepine and cancer risk in humans. However, in our study, a significant inverse association between carbamazepine and colorectal cancer was found in analyses of the FAERS and JMDC claims databases. This result suggested that carbamazepine may also be a potential anticancer drug candidate. Further study is required to confirm this finding.

For lamotrigine and topiramate, significant inverse associations were found for several cancers only in the analysis of the FAERS, but not the JMDC, database. To date, there has been no report regarding the association between these AEDs and cancer risk. In this study, the detection of significant inverse signals from analyses of both databases was applied as a strict criterion for defining significant associations. Consequently, associations between sodium channel-blocking AEDs and these cancers are unclear. Further studies are required to evaluate whether these AEDs reduce the risk of cancer.
Although the analysis of spontaneous reports is a useful method for identifying signals, there are several potential limitations that should be taken into account when interpreting results obtained from spontaneous reporting databases. First, there is no certainty that the reported event was actually due to the drug. Second, not every adverse event or medication error that occurs with a drug product is reported. Third, the database is missing data and has frequent misspellings of drug names. Fourth, no individual algorithm is adequate to detect signals, and the concurrent use of other algorithms is essential. Therefore, ROR and IC algorithms were used in the analysis of the FAERS database, and the adverse events were listed as drug-associated when the two indices met the criteria in the current study. Furthermore, in the current study, a different methodology, ESSA of the JMDC claims database, was used to confirm the findings of the FAERS database analyses. Of course, the ESSA is associated with several potential limitations due to its application to a claims database. First, our study population was selected from beneficiaries covered by the employees' health insurance system. Because most beneficiaries are working adults or their family members, the proportion of elderly patients aged $\geq 65$ years is low. Second, the diagnoses listed in the claims were not validated. We generally needed to consider the diagnosis contained in the claim, which is listed for health insurance claims. However, it is obvious that serious diseases such as cancer may not be listed in the claim only for the purpose of health insurance claims; that is, the patient is likely to actually have the disease. Third, individual cases were not reviewed, and other causes were not considered. Potential confounding factors, including smoking history, health history, race/ethnicity, body mass index and occupation, which are associated with cancer, could not be controlled in this study. Lack of data on these potential confounding factors should be considered as a limitation when interpreting our findings. Mean age of antiepileptic drug users identified in the study was younger compared to the common cancer patients. Advanced age is the most important risk factor for cancer. Therefore, study patients for ESSA may be less likely to develop cancer. However, the ESSA is based on within-subject comparisons, and this method allows patients to serve as their own comparator. Therefore, the ESSA is similar to the case-crossover design, in which exposures during a fixed period before the case date (date when the target outcome occurred) and prior dates were compared in the same individual. These within-subject comparisons can thus be fully controlled for potential confounding between-subject differences and time-invariant characteristics, including age, gender, 
genetic factors, mental health status, and other unknown confounding factors $[44,45]$.

Although these potential limitations should be taken into account when interpreting the results obtained from this study, it is noteworthy that the multi-methodological approaches used, comprising different algorithms and databases, detected significant inverse associations between sodium channel-blocking AEDs and certain cancers. Disproportionality and symmetry analyses were originally developed to detect unknown adverse events for pharmacovigilance. We applied these methodologies and algorithms to the detection of inverse signals of cancer associated with sodium channel-blocking AED use, and our study detected inverse associations between sodium channel-blocking AED use and several cancers. If these inverse signals reveal unknown effects of sodium channel-blocking AED, these methodologies may be potential tools to detect unknown clinical effects. Further studies are required to validate or evaluate the potential of these methodologies.

\section{Conclusions}

Multi-methodological approaches using different methodologies, algorithms, and databases suggest that sodium channel-blocking AED use is inversely associated with several cancers. Although a number of experimental studies suggest that sodium channel-blocking AEDs are potential candidates for anti-cancer agents, no definitive evidence exists. The present study demonstrated an inverse association between sodium channel-blocking AED use and the risk of certain cancers. Further epidemiological and observational studies are required to confirm our findings. In addition, further studies are required to verify the hypothesis that multi-methodological approaches using different methodologies, algorithms, and databases may be useful tools to explore unknown clinical effects of drugs.

\section{Abbreviations}

VGSC: Voltage-gated sodium channel; AED: Antiepileptic drugs; FAERS: FDA Adverse Event Reporting System; FDA: Food and Drug Administration; MedDRA: Medical Dictionary for Regulatory Activities; SMQ: Standardized MedDRA Queries; PT: preferred term; ROR: reporting odds ratio; IC: information component; JMDC: The Japan Medical Data Center; ICD-10: International Classification of Diseasse, 10th Revision; ESSA: Event sequence symmetry analysis; SR: Sequence ratio.

\section{Acknowledgements}

The authors thank the Japan Medical Data Center Co., Ltd for providing the claims database information.

\section{Competing Interests}

The authors have declared that no competing interest exists.

\section{References}

1. Mantegazza M, Curia G, Biagini G, et al. Voltage-gated sodium channels as therapeutic targets in epilepsy and other neurological disorders. The Lancet Neurology. 2010; 9: 413-424.

2. Black JA, Waxman SG. Noncanonical roles of voltage-gated sodium channels. Neuron. 2013; 80: 280-291.

3. Brackenbury WJ, Djamgoz MB. Activity-dependent regulation of voltage-gated $\mathrm{Na}+$ channel expression in Mat-LyLu rat prostate cancer cell line. The Journal of physiology. 2006; 573: 343-356.

4. Brackenbury WJ. Voltage-gated sodium channels and metastatic disease. Channels (Austin, Tex). 2012; 6: 352-361.

5. House CD, Vaske CJ, Schwartz AM, et al. Voltage-gated Na+ channel SCN5A is a key regulator of a gene transcriptional network that controls colon cancer invasion. Cancer research. 2010; 70: 6957-6967.

6. Fraser SP, Diss JK, Chioni AM, et al. Voltage-gated sodium channel expression and potentiation of human breast cancer metastasis. Clinical cancer research : an official journal of the American Association for Cancer Research. 2005; 11: 5381-5389.

7. Grimes JA, Fraser SP, Stephens GJ, et al. Differential expression of voltage-activated $\mathrm{Na}+$ currents in two prostatic tumour cell lines: contribution to invasiveness in vitro. FEBS letters. 1995; 369: 290-294.

8. Brackenbury WJ, Chioni AM, Diss JK, et al. The neonatal splice variant of Nav1.5 potentiates in vitro invasive behaviour of MDA-MB-231 human breast cancer cells. Breast cancer research and treatment. 2007; 101: 149-160.

9. Brackenbury WJ, Djamgoz MB. Nerve growth factor enhances voltage-gated $\mathrm{Na}+$ channel activity and Transwell migration in Mat-LyLu rat prostate cancer cell line. Journal of cellular physiology. 2007; 210: 602-608.

10. Abdul M, Hoosein N. Inhibition by anticonvulsants of prostate-specific antigen and interleukin- 6 secretion by human prostate cancer cells. Anticancer research. 2001; 21: 2045-2048.

11. Abdul M, Hoosein N. Voltage-gated sodium ion channels in prostate cancer: expression and activity. Anticancer research. 2002; 22: 1727-1730.

12. Brisson L, Gillet L, Calaghan $\mathrm{S}$, et al. $\mathrm{Na}(\mathrm{V}) 1.5$ enhances breast cancer cell invasiveness by increasing NHE1-dependent $\mathrm{H}(+)$ efflux in caveolae. Oncogene. 2011; 30: 2070-2076.

13. Yang M, Kozminski DJ, Wold LA, et al. Therapeutic potential for phenytoin: targeting $\mathrm{Na}(\mathrm{v}) 1.5$ sodium channels to reduce migration and invasion in metastatic breast cancer. Breast cancer research and treatment. 2012; 134: 603-615.

14. Fairhurst C, Watt I, Martin F, et al. Exposure to sodium channel-inhibiting drugs and cancer survival: protocol for a cohort study using the QResearch primary care database. BMJ open. 2014; 4: e006604

15. Sakaeda T, Tamon A, Kadoyama K, et al. Data mining of the public version of the FDA Adverse Event Reporting System. International journal of medical sciences. 2013; 10: 796-803.

16. Almenoff JS, Pattishall EN, Gibbs TG, et al. Novel statistical tools for monitoring the safety of marketed drugs. Clinical pharmacology and therapeutics. 2007; 82: 157-166.

17. van Puijenbroek EP, Bate A, Leufkens HG, et al. A comparison of measures of disproportionality for signal detection in spontaneous reporting systems for adverse drug reactions. Pharmacoepidemiology and drug safety. 2002; 11: 3-10.

18. Bate A, Lindquist M, Edwards IR, et al. A Bayesian neural network method for adverse drug reaction signal generation. European journal of clinical pharmacology. 1998; 54: 315-321.

19. Kimura S, Sato T, Ikeda S, et al. Development of a database of health insurance claims: standardization of disease classifications and anonymous record linkage. Journal of epidemiology / Japan Epidemiological Association. 2010; 20: 413-419.

20. Hallas J. Evidence of depression provoked by cardiovascular medication: a prescription sequence symmetry analysis. Epidemiology (Cambridge, Mass). 1996; 7: 478-484.

21. Tsiropoulos I, Andersen M, Hallas J. Adverse events with use of antiepileptic drugs: a prescription and event symmetry analysis. Pharmacoepidemiology and drug safety. 2009; 18: 483-491.

22. Hallas J, Gaist D, Bjerrum L. The waiting time distribution as a graphical approach to epidemiologic measures of drug utilization. Epidemiology (Cambridge, Mass). 1997; 8: 666-670. 
23. Morris JA, Gardner MJ. Calculating confidence intervals for relative risks (odds ratios) and standardised ratios and rates. British medical journal (Clinical research ed). 1988; 296: 1313-1316.

24. Nelson M, Yang M, Dowle AA, et al. The sodium channel-blocking antiepileptic drug phenytoin inhibits breast tumour growth and metastasis. Molecular cancer. 2015; 14: 13.

25. Fraser SP, Salvador V, Manning EA, et al. Contribution of functional voltage-gated $\mathrm{Na}+$ channel expression to cell behaviors involved in the metastatic cascade in rat prostate cancer: I. Lateral motility. Journal of cellular physiology. 2003; 195: 479-487.

26. Bug G, Ritter M, Wassmann B, et al. Clinical trial of valproic acid and all-trans retinoic acid in patients with poor-risk acute myeloid leukemia. Cancer. 2005; 104: 2717-2725.

27. Minucci S, Pelicci PG. Histone deacetylase inhibitors and the promise of epigenetic (and more) treatments for cancer. Nature reviews Cancer. 2006; 6: 38-51.

28. Deroanne CF, Bonjean $\mathrm{K}$, Servotte $\mathrm{S}$, et al. Histone deacetylases inhibitors as anti-angiogenic agents altering vascular endothelial growth factor signaling. Oncogene. 2002; 21: 427-436.

29. Kim MS, Kwon HJ, Lee YM, et al. Histone deacetylases induce angiogenesis by negative regulation of tumor suppressor genes. Nature medicine. 2001; 7: 437-443.

30. Johannessen CU, Johannessen SI. Valproate: past, present, and future. CNS drug reviews. 2003; 9: 199-216.

31. Gottlicher M, Minucci S, Zhu P, et al. Valproic acid defines a novel class of HDAC inhibitors inducing differentiation of transformed cells. The EMBO journal. 2001; 20: 6969-6978.

32. Catalano MG, Fortunati N, Pugliese M, et al. Valproic acid induces apoptosis and cell cycle arrest in poorly differentiated thyroid cancer cells. The Journal of clinical endocrinology and metabolism. 2005; 90: 1383-1389.

33. Takai N, Desmond JC, Kumagai T, et al. Histone deacetylase inhibitors have a profound antigrowth activity in endometrial cancer cells. Clinical cancer research : an official journal of the American Association for Cancer Research. 2004; 10: 1141-1149.

34. Takai N, Kawamata N, Gui D, et al. Human ovarian carcinoma cells: histone deacetylase inhibitors exhibit antiproliferative activity and potently induce apoptosis. Cancer. 2004; 101: 2760-2770.

35. Chavez-Blanco A, Perez-Plasencia C, Perez-Cardenas E, et al. Antineoplastic effects of the DNA methylation inhibitor hydralazine and the histone deacetylase inhibitor valproic acid in cancer cell lines. Cancer cell international. 2006; 6: 2.

36. Mottamal M, Zheng S, Huang TL, et al. Histone deacetylase inhibitors in clinical studies as templates for new anticancer agents. Molecules (Basel, Switzerland). 2015; 20: 3898-3941.

37. Berendsen S, Broekman M, Seute T, et al. Valproic acid for the treatment of malignant gliomas: review of the preclinical rationale and published clinical results. Expert opinion on investigational drugs. 2012; 21: 1391-1415.

38. Blaheta RA, Michaelis M, Driever PH, et al. Evolving anticancer drug valproic acid: insights into the mechanism and clinical studies. Medicinal research reviews. 2005; 25: 383-397.

39. Tan J, Cang S, Ma Y, et al. Novel histone deacetylase inhibitors in clinical trials as anti-cancer agents. Journal of hematology \& oncology. 2010; 3: 5.

40. Bilen MA, Fu S, Falchook GS, et al. Phase I trial of valproic acid and lenalidomide in patients with advanced cancer. Cancer chemotherapy and pharmacology. 2015

41. Chu BF, Karpenko MJ, Liu Z, et al. Phase I study of 5-aza-2'-deoxycytidine in combination with valproic acid in non-small-cell lung cancer. Cancer chemotherapy and pharmacology. 2013; 71: 115-121.

42. Bauman J, Shaheen M, Verschraegen CF, et al. A Phase I Protocol of Hydralazine and Valproic Acid in Advanced, Previously Treated Solid Cancers. Translational oncology. 2014; 7: 349-354.

43. Kang H, Gillespie TW, Goodman M, et al. Long-term use of valproic acid in US veterans is associated with a reduced risk of smoking-related cases of head and neck cancer. Cancer. 2014; 120: 1394-1400.

44. Vegter S, de Boer P, van Dijk KW, et al. The effects of antitussive treatment of ACE inhibitor-induced cough on therapy compliance: a prescription sequence symmetry analysis. Drug safety : an international journal of medical toxicology and drug experience. 2013; 36: 435-439.

45. Lai EC, Yang YH, Lin SJ, et al. Use of antiepileptic drugs and risk of hypothyroidism. Pharmacoepidemiology and drug safety. 2013; 22: 1071-1079. 